\title{
La inserción laboral de los jóvenes: un concepto histórico, ambiguo y societal $^{1}$
}

\section{Professional integration of youth: A historical, ambiguous and societal concept}

Éric Verdier

Laboratoire d'économie et de sociologie du travail du CNRS, Francia

eric.verdier@uni-amu.fr

Mircea Vultur

Centre Urbanisation, Culture, Société de l'INRS, Canadá

mircea.vultur@ucs.inrs.ca

\section{Resumen:}

Los conceptos principales que se utilizan hoy en día para analizar la situación en el mercado laboral de las diferentes categorías de fuerza de trabajo son a menudo heredados del periodo de los Trente Glorieuses ${ }^{2}$ ( $\mathrm{N}$ de la T: Edad de oro del capitalismo, en español). Un concepto que se convirtió en un objeto de investigación, que varios trabajos trataron de circunscribir y de definir de varias maneras, es el de inserción laboral. Los objetivos de este artículo son: a) mostrar el carácter histórico de la noción de inserción laboral, b) analizar la ambigüedad de sus definiciones proteiformes, utilizadas en gran parte por los investigadores en función de sus preocupaciones específicas de investigación y c) resaltar el aspecto societal de la inserción laboral a partir de una mirada comparativa sobre el contenido de esta concepción y de su utilización en Francia y en Quebec. Este proceso nos llevará a un amplio cuestionamiento sobre la adecuación de este concepto a la realidad de los recorridos ${ }^{3}$ de formación y del mundo del trabajo contemporáneos que sufren profundas y rápidas transformaciones y requieren nuevas conceptualizaciones más detalladas y más rigurosas.

Palabras CLAVE: Inserción laboral de los jóvenes, Debate teórico, Análisis societal, Acción pública, Renovación conceptual.

\section{Abstract:}

Many of the major concepts currently used to analyze market labour market conditions for various categories of workers are inherited from the Trente Glorieuses\#the thirty-year period following the end of the Second World War in France. Professional integration is one example that has frequently drawn the attention of researchers attempting to define it in various ways. This article aims to: a) reveal the historical nature of the concept of professional integration; b) analyze the ambiguity created by its multifaceted definitions, which researchers tend to variously adopt depending on their specific research interests; and c) highlight the societal aspect of professional integration, through a comparative study of the meanings assigned to the concept and of how it is used in France and Quebec. This approach raises broader questions about the suitability of the concept for analyzing contemporary realities. Both educational pathways and the world of work are undergoing profound and rapid changes, creating a need for more nuanced and rigorous conceptualizations.

KEYWORDS: Professional integration of youth, The oretical debate, Societal analysis, Public action, Conceptual renewal.

\section{INTRODUCCIÓN}

La problemática de la inserción, pensada como modo de la integración ala sociedad, encuentra sus bases en los trabajos de la Escuela de Chicago, que desarrolló teóricamente este concepto dentro del contexto de los Estados Unidos en el periodo de entreguerras, trabajos que debieron afrontar los desafíos de asimilar sus minorías étnicas, como consecuencias de varios sedimentos migratorios (Coulon, 2012). El surgimiento del American wayoflife impuso una concepción de la integración a la sociedad, gracias al trabajo y a las posibilidades de aprobación social que este estilo de vida ofrecía basado en la adhesión a valores comunes que no eran tributarios de las consideraciones del Estado ni de las políticas sociales. En las sociedades

Recepción: 14 de noviembre de 2018 | Aprobación: 12 de diciembre de 2018 | Publicación: 4 de enero de 2019

Cita sugerida: Verdier, E. y Vultur, M. (2018). La inserción laboral de los jóvenes: un concepto histórico, ambiguo y societal. Cuestiones de Sociología, 19, e067. https://doi.org/10.24215/23468904e067 
asalariadas contemporáneas, particularmente en Europa, el campo de investigación de la inserción laboral se desarrolló ulteriormente junto con la prolongación del tiempo de formación de los jóvenes y con sus dificultades de acceso a empleos estables. Estos fenómenos aparecieron al término de los Trente Glorieuses, periodo durante el cual "el ingreso al trabajo no era un problema y la inserción tampoco era una categoría pertinente"(Dubar, 2001, p. 24). Luego, esto cambió debido a la convergencia de dos "rupturas históricas". La primera tiene que ver, en muchos países (con excepción notable de los países germánicos donde el aprendizaje sigue predominando), con la disyunción entre el espacio específico de formación (instrucción y educación) y el espacio de actividad de trabajo (empleo e ingresos) que contribuyó a poner de manifiesto una nueva era de la vida, "la juventud". La segunda, más reciente, hace referencia a la disociación entre la culminación de los estudios y el ingreso al empleo, consecuencia de fenómenos como el desempleo en masa, los empleos atípicos y precarios, la desvalorización escolar y la sobre cualificación laboral. "Por cierto, una coyuntura histórica determinada que constituye el contexto del surgimiento del problema social de la inserción de los jóvenes" (Dubar, 2001, p. 26). Hoy en día, la situación en el mercado laboral, los modos de acceso al empleo y el rapport ${ }^{4}$ en el trabajo de los jóvenes se transformaron significativamente y suscitan, en particular en los países desarrollados, un debate legítimo sobre la actualidad y la pertinencia del concepto de inserción laboral de los jóvenes. En esta perspectiva sociohistórica, este artículo propone abordar los lazos complejos y evolutivos entre las categorizaciones de la inserción laboral provenientes de la acción pública, presentadas por el análisis estadístico, y finalmente las categorizaciones producidas en el campo académico: a) mostrando el carácter histórico de este concepto, b) analizando la ambigüedad de sus definiciones proteiformes, utilizadas en gran parte por los investigadores en función de sus preocupaciones específicas de estudio y c) haciendo hincapié en el aspecto societal de la inserción laboral a partir de una mirada comparativa de su contenido en Francia y en Quebec. Este procedimiento nos llevará a un cuestionamiento más amplio sobre la capacidad de este concepto que da cuenta de la realidad de los recorridos de formación y del mundo del trabajo contemporáneos que sufren profundas y rápidas transformaciones y que necesitan nuevas conceptualizaciones.

\section{Regreso a LA HiSTORIA DE UN CONCEPTO: ENTRE NORMAS SOCIALES Y CATEgORÍAS DE LA ACCIÓN PÚBLICA}

Históricamente, el concepto de inserción laboral desarrollado en Francia es el resultado de dos matrices del Estado social a la francesa. La primera matriz, de la cual proviene incluso el concepto de inserción\#que cubre, hoy como ayer, un campo mucho más amplio que el que se refiere a los jóvenes\# se desarrolló en los años '60, en el marco de políticas públicas que pretendían facilitar el acceso de las personas con discapacidad al empleo y a recursos pecuniarios regulares (Guitton, 1998). Este modelo de la inserción mediante el trabajo se inspiró y se distinguió a la vez de la política de integración laboral de las personas con discapacidad. Encontramos el mismo voluntarismo político que rechaza la asistencia y la indemnización pasiva de los riesgos sociales (discapacidad versus desempleo) y que, por lo tanto, apela a la movilización de las fuerzas de la sociedad civil que buscan promover nuevas solidaridades activas (Eme, 2012). Esta filiación se manifiesta también en "el carácter derogatorio y a menudo precario de los estatus de actividad propuestos a los beneficiarios" que no hacen tanta referencia "a un imperativo de flexibilidad como a la transposición del esquema teórico de la transición” (Eme, 2012, p, 25) en vigencia para las personas con discapacidad. Siguiendo esta matriz, el concepto de inserción laboral se aplicará mayoritariamente a públicos considerados como desfavorecidos con respecto a posibilidades de acceso al empleo e identificados con respecto al sexo, al nivel de formación o de cualificación, o incluso, a las distintas características (estatus de inmigración, discapacidad, etc.). La segunda matriz que da origen al concepto de inserción laboral está relacionada con el despliegue de la planificación a la francesa (Tanguy, 2002) con el fin de asegurar una adecuación de la formación laboral, que tiene como objetivo satisfacer las importantes necesidades en cualificaciones de las empresas generadas por el fuerte crecimiento económico que prevalecía durante el 
periodo de Trente Glorieuses. Luego, la acción pública no ha dejado de desarrollar dispositivos de inserción laboral, incluso dentro de la propuesta de formación inicial, es decir, la profesionalización de los estudios se impone como una técnica mayor en un contexto de crisis recurrente del trabajo, que afecta, ante todo, a los jóvenes.(Charles, 2015).

\subsection{Primera matriz: de la integración a la inserción laboral}

El modelo de la inserción mediante el trabajo se afirma al superar a la matriz de la integración que prevaleció por mucho tiempo. Si bien, innegablemente, la noción de integración encuentra un lugar destacado en un árbol genealógico de la categoría de acción pública de la inserción, esta última suplanta la primera porque resulta ser, política y técnicamente, un concepto claramente más flexible y eficaz que el de integración, proveniente de la sociología durkheimiana y notoriamente impulsado por el desarrollo de la precarización, y de la "desafiliación" (Castel, 1995), que afectan particularmente a los jóvenes. Pero el reemplazo del concepto de integración, relacionado con un modelo social definido a través de un Estado dominante, por el de inserción, significa igualmente el surgimiento de un nuevo modus operandi de las políticas sociales que procura basar el acceso a la ciudadanía social no solo en ocupaciones de cargos institucionales sino igualmente en las conductas de los mismos individuos, que deben ser actores explícitos de la conquista de su autonomía. Por lo tanto, el éxito de este proceso depende tanto de la calidad y de la eficacia de las prácticas de acompañamiento por profesionales de la inserción como de las actitudes de los jóvenes, con dificultades en el mercado laboral, a quienes se debe inculcar "un hábito flexible, responsable y autónomo", que marque la prevalencia de la "lógica económica" sobre la "lógica educativa" (Mauger, 2001, p. 13). De manera sigilosa al menos, esta "activación de los individuos" (Barbier, 2002) introduce la posibilidad de una transferencia parcial de responsabilidad hacia los intereses, en este caso los jóvenes, con respecto a su futuro ${ }^{5}$. Así, constatamos que el concepto de inserción laboral está íntimamente relacionado con el concepto más amplio de inserción social, "destinado a promover individuos autónomos, responsables de sí mismos, que en un principio, en los años sesenta, eran vulnerables y a menudo considerados como inadaptados. De esta manera ellos llegan a ser verdaderos ciudadanos." (Bonniel y Lahire, 1994, p. 26).

En Francia, le rapport Schwartz (1981)(el informe Schwartz, en español), dedicado a brindar soluciones a las "dificultades de inserción social y laboral de los jóvenes", proporciona un nuevo referencial de acción pública (Jobert y Muller, 1987), que va luego a desplegarse en varios registros, alejándose en ocasiones del sentido original. Estos registros incluyen dispositivos, que articulan, en forma de contratos de trabajo de tipo particular, formación y empleo, con el objetivo de obtener: una calificación a través de los jóvenes que salen del sistema educativo sin diploma; medidas públicas que pretenden ubicar a los jóvenes en empresas para dotarlos de una primera experiencia laboral, no bajo la categoría socioprofesional de empleado, sino de practicante rentado, atribuyéndole el pago de una indemnización pública; actividades de utilidad social convocadas a desarrollarse en el sector sin fines de lucro (colectividades territoriales, establecimientos públicos, asociaciones). Es así como la inserción de los jóvenes se convierte en un "asunto de Estado" (Verdier, 1995). Este último está destinado a combatir el desempleo juvenil con la ayuda de un arsenal de medidas aún más diversificadas dado que cada gobierno que critica la acción de su (sus) predecesor (es) reforma los dispositivos existentes, particularmente agregando nuevos. De esta manera se desarrolla un espacio sociopolítico de la inserción que está dotado de una cosmogonía cada vez más compleja de profesionales que defienden el mantenimiento de sus misiones en nombre del interés de los jóvenes en dificultad que ellos acompañan y orientan (Mauger, 2001). 


\subsection{Segunda matriz: planificar para construir la adecuación formación/empleo}

El modelo del Estado "ingeniero de lo social" (Desrosiè res, 2003), que debe responder a las necesidades de las empresas e inculcar a los jóvenes un cierto ethos y actitudes en conformidad con las normas dominantes del mundo del trabajo, se apoyó, en la segunda mitad de los años '60, en la creación y el desarrollo de un conjunto de "administradores expertos"(Massardier, 1996) que van a respaldar técnicamente una planificación concertada, organizadora de la adecuación formación/empleo. En este aspecto, en 1969 en Francia, la creación de la nomenclatura de los niveles de formación constituye un momento clave; siempre vigente, este instrumento pone en correspondencia los dos "mundos", bien distintos, que son el sistema educativo y el mercado laboral. Es así como, bajo el auspicio del Institutnational de la statistique et des études économiques(INSEE) (Instituto Nacional de Estadística y Estudios Económicos, en español), los balances formación/empleo van a respaldar durante los años'70, previsiones cuantificadas destinadas a integrar, en una única herramienta prospectiva, las diferentes modalidades de renovación de la fuerza de trabajo, juvenil y adulta, a los diversos niveles de cualificación. Por consiguiente, es necesario señalar una doble dinámica, institucional y cognitiva, que en aquel momento genera la planificación a la francesa; en esa coyuntura, en marzo de 1970, se crea el Centre d'études et de recherches sur les qualifications(CÉREQ) (Centro de estudios y de investigaciones sobre las cualificaciones, en español) responsable de elaborar las perspectivas del Ministerio de la Educación Nacional como parte de la concertación nacional dirigida por la Commissariat général du plan (Comisión general del Plan, en español) y además, responsable de aportar su contribución a la vinculación previsional de la formación y del empleo que coordina la INSEE. El CÉREQ realiza investigaciones de inserción que, a mitad de los años '70, van a inscribirse en un dispositivo permanente, el Observatoire national des entrées dans la vie active (ONEVA) (Observatorio nacional de las entradas en la vida activa, en español). Su recurso clave estará constituido de investigaciones(retrospectivas) de recorridos laborales que consiste, para cada nivel de formación, en investigar, durante varios años, la inserción en el empleo de quienes salen del sistema educativo y todo esto, para las diferentes especialidades profesionales.

\subsection{Panorama sobre el surgimiento del tema de la inserción en Quebec}

En Quebec, la cuestión de la inserción laboral surge como el resultado de la importancia que toman los jóvenes en la orientación de la sociedad durante la "Revolución Tranquila" (Rioux, 1969; Gauthier, 2007). Por lo tanto, los análisis que produce tienen como referente el enfoque de inspiración francesa, desarrollado en un contexto muy diferente. Las representaciones sociales y las prácticas de los jóvenes no correspondían, como en Francia, a trayectos lineales de la escuela al empleo complementados con identificaciones estatutarias simples. El tema de la inserción se planteaba particularmente en periodos de crisis económicas (Gauthier, 2001; Hamel, 2007), "para dar nuevas bases a una dinámica societal [...] donde el empleo pleno era visto como la única vía de inserción social y de reconocimiento del valor de los individuos” (Saint-Pierre, 2001, p. 68). Esta cuestión requería acciones políticas a favor de los jóvenes, lo que implicaba conocer su situación en el mercado laboral. Por ello, a partir de 1970 se crearon organismos de observación de la inserción laboral tanto en Quebec como en la totalidad de Canadá. A diferencia de Francia, estos organismos son muy descentralizados y realizan investigaciones en diversos niveles de gobierno (Moulin y Doray, 2007): federal, provincial y local (instituciones de educativas). Son el reflejo del funcionamiento del sistema de enseñanza que, en Canadá, corresponde al nivel de las provincias; el gobierno federal lo sostiene, sobre todo en el plano de la investigación, con miras a favorecer el desarrollo económico. En este contexto, a nivel provincial quebequense, se creó en los años'70 una herramienta estadística de observación de la situación de los jóvenes en el mercado laboral luego de su graduación, las encuestas Relances, realizadas de manera regular en el nivel secundario, collégial ${ }^{6}$ y universitario. Estas se completan mediante encuestas específicas realizadas a 
cada establecimiento que generan datos sobre la situación de sus propios graduados. El Ministerio de Trabajo de Quebec realiza también investigaciones puntuales sobre la situación de los jóvenes que participaron en diversas políticas de empleabilidad. A nivel federal, surge a fines de los años' 90 un nuevo dispositivo, la enquête sur les jeunes en transition (EJET) (encuesta a los jóvenes en transición, en español), dirigida a una "población de referencia que no queda definida por el año de finalización de los estudios de jornada completa, ni tampoco por el año de obtención de los diplomas, sino simplemente por la edad" (Moulin, 2011, p. 66), en este caso, 15 años para una cohorte, 16-18 años para la otra, registradas a lo largo del tiempo en forma de datos de panel lo que da lugar a relevamientos regulares, sin que haya lazo explícito con el despliegue de tal o cual instrumento de acción pública, como sí lo fue en el caso en Francia.

\section{LA INSERCIÓN LABORAL, ¿UN OBJETO VAGO QUE ESCAPA DE LA TEORÍA?}

Ya sea con respecto a la definición del concepto o a su operación alización en las investigaciones empíricas, la inserción laboral sigue siendo un objeto vago que parece escapar de la teoría. Como lo comprueba Dubar en 1998, "las investigaciones sociológicas en el ingreso de los jóvenes a la vida activa, y especialmente en la transición de la escuela al mercado laboral, permitieron recopilar conocimientos empíricos diversos y relativamente acumulativos sin por ello hacer de la inserción un concepto preciso, relacionado con una teoría identificable" (p. 30). Bourdon y Cleaver (2000) muestran igualmente que "la inserción laboral es un fenómeno que, si fue y es a menudo estudiado, está aún lejos de ser el objeto de una teorización coherente, ampliamente aceptada" (p. 677).

Es necesario decir que hace ya 30 años, Tanguy (1986) notaba la complejidad de la inserción "debido al desarrollo de las formaciones en alternancia [...] que multiplican los estatus particulares" (p. 68). Luego, la diversidad de los itinerarios de inserción para un mismo tipo de diploma, objeto de la investigación de Paul (1989), fue reiteradamente señalada: muchos estudios concluyen en "la desintegración de los trayectos de inserción de los jóvenes", bajo la influencia de lo que Lichtenberger (1995) califica como "alternancia salvaje" entre las diversas formas de empleo, el desempleo recurrente y los dispositivos de ayuda a la inserción que multiplicaron las políticas públicas de empleo. Además, la descentralización de la formación profesional suscitó estudios sobre las "disparidades regionales" que dan testimonio de la importancia del contexto económico mientras que los contrastes locales traducen las dinámicas de los mercados locales de trabajo y los modos de organización de los "sistemas localizados de inserción" (Demazière et Dubar, 1993, p.18).

La literatura sobre la inserción estará igualmente marcada por una suerte de carrera de persecución entre, por un lado, la necesidad de multiplicar los estudios empíricos para estar en condiciones comprender la diversificación creciente de los fenómenos sociales en cuestión y, por otro lado, la necesidad de disponer de un entramado teórico en condiciones de volver inteligible el conjunto de las investigaciones empíricas acumuladas. Resulta entonces una doble constatación recurrente, de una complejidad empírica creciente y de una conceptualización de la inserción incesantemente rebasada por la acumulación de análisis, que aluden a los modos juveniles de acceso al empleo cada vez más diversos. Además, el desarrollo del desempleo de los jóvenes que, en casi todas las sociedades supera al de los adultos, perjudica tanto los esquemas interpretativos como los objetivos políticos vehiculizados por las dos matrices originales de la inserción: el proyecto voluntarista de acceso al empleo de los jóvenes menos cualificados ¿no está profundamente desestabilizado por la sobreexposición constante de estos últimos al desempleo? Por su parte, el modelo adecuacionista, proveniente de la planificación, ¿no es cuestionado por la prolongación constante del "sas"7 que constituye la inserción laboral? Estas tensiones, tanto interpretativas como políticas alcanzan dos dimensiones claves de la inserción: la medición y el análisis del proceso. 


\section{1 ¿ Se puede medir la inserción?}

Autores como Bonniel y Lahire (1994, p. 21) niegan aún la posibilidad de medir la inserción: "Sería absurdo pretender medir científicamente el nivel de inserción [...] a partir de definiciones arbitrarias y nunca objetivas (aunque estas contengan todas las características aparentes) que solo suelen retomar implícitamente una definición particular socialmente aceptada". No obstante, sin duda, el desarrollo de un dispositivo estadístico ad hoc se tradujo en la elaboración de definiciones de la inserción en términos de indicadores usuales con respecto al acceso al mercado laboral, la exposición al desempleo, el tipo y el número de empleos ocupados por un individuo desde su salida del sistema educativo, la duración de acceso a un primer trabajo, el tipo de correspondencia entre la formación adquirida y los puestos ocupados, etc. Sabemos con Desrosiè res (2008) que la estadística no se satisface con abarcarlo real, sino que contribuye a instituirlo. Así, el primer riesgo al cual se exponen estos indicadores, como componentes cuantificables de la inserción laboral, consiste en difundir un enfoque estadístico de un fenómeno fundamentalmente procesual. En cuanto a lo dicho, la relación entre de los diplomas obtenidos y la tasa de desempleo de sus titulares medida en un cierto plazo-generalmente algunos meses después de la obtención del título- o bien del salario medio del primer empleo, son los síntomas más frecuentes de esta derivación. Efectivamente, proceder a dichas evaluaciones es probablemente difícil de evitar, principalmente porque hacen posible comparaciones cuantificadas en el espacio y en el tiempo, y porque la comunicación política así como el debate público se resisten a ser ignorados. Pero, además del simplismo reductor de la inserción, esta representación de la inserción laboral puede suscitar una lectura de las dificultades de acceso al empleo como consecuencia de una propuesta: la de los jóvenes y de los padres en razón de sus supuestas malas elecciones de orientación y la de los formadores cuya calidad de oferta sería insuficiente para dar respuesta a un rápido acceso laboral. Ciertamente, tales factores pueden contribuir, pero no podrán ser los únicos determinantes: estos interactúan siempre en mayor o menor medida con las formas de gestión de las empresas y la coyuntura macroeconómica del empleo, apenas tomadas en cuenta por tales indicadores. Fundamentalmente, tal aproximación desconoce interrogantes sin embargo cruciales: ¿en qué momento comienza la inserción de un joven? ¿al salir del sistema educativo? ¿al término de la escolaridad obligatoria? ¿durante sus primeras experiencias en el mercado laboral que pueden ocurrir durante los estudios? Además, ¿cuándo se considera que la inserción del individuo está terminada? ¿cuándo la persona obtiene un empleo estable?¡cuándo la persona se considera estabilizada en el mercado laboral? Tantas preguntas cuyos parámetros de referencia no son fáciles de establecer y que, por consiguiente, encuentran en los trabajos de investigación respuestas que, pese a esfuerzos conceptuales indiscutibles, no dejarán de aparecer fluctuantes.

\subsection{Balances dubitativos}

Desde los años '80, Tanguy (1986), realizó “un estado de las investigaciones en Francia” sobre la relación formación/empleo. En ese trabajo, el autor no solo calificaba esta relación de "inencontrable" sino que, además, señalaba la ausencia de una verdadera teoría de la inserción, cuyas razones se deberían tanto a la fuerte dependencia de la investigación con respecto a los encargos de la administración económica y social como a la excesiva predominancia -en el campo de las ciencias sociales- de los trabajos de los economistas, que habrían mostrado una "tendencia a olvidar que la inserción es el reflejo [...] del modo de funcionamiento del mercado laboral” (Tanguy, 1986, p. 87). Por su parte, la sociología habría sido caracterizada por "enfoques temáticos que siguen siendo múltiples [que solo podríamos] reagrupar artificialmente dentro de una sociología de la inserción” (Tanguy, 1986, p. 88). Prolongando balances posteriores a estos análisis, Bouffartigue, Lagrée y Rose (1989), Plottier (1990) y Bordigoni, Demazière y Mansuy (1994) ven en la falta de teorización de la inserción laboral de los jóvenes la confirmación de la influencia excesiva de los "encargos institucionales" cuyas "categorías" actuarían como "figuras impuestas" 
(Bordigono y colaboradores, 1994, p. 7). Sin embargo, estos autores notan que este campo de investigación se independizó al punto de encontrar su propia base gracias a la realización de varias investigaciones tanto económicas como sociológicas; al respecto concluyen que, en adelante, el tema de la inserción es "construido y problematizado de manera multiforme, con referencia a marcos teóricos y a metodologías diversificadas" (p. 7). En el mismo periodo, Nicole-Drancourt y Roulleau-Berger (1995) se muestran claramente más pesimistas - "la inserción [sigue siendo] un objeto de investigación en construcción" porque "medir la inserción [es] un proyecto difícil" (p. 41)- antes de señalar que el "marco de análisis de la inserción" [es siempre] "inencontrable” (p. 43), debido a la multiplicidad de las problemáticas y de las aproximaciones que permiten abordar este tema. A comienzos del año 2000, la inserción laboral es incluso calificada por Giretcomoun "concepto proteiforme" (Giret, 2000) mientras que Dubar destaca que "la construcción de las investigaciones sobre la inserción [es] en sí misma un proceso amplio y complejo que [está] lejos de tener el marco teórico que permite volverlo acumulativo” (Dubar, 2001, p. 35).

\section{3 ¿Cuándo podemos decir que la inserción se termina?}

Sin embargo, una serie de trabajos desarrollados en la transición de los años '90-2000, produjo avances notables, particularmente buscando responder a una pregunta simple de formular, pero cuyas respuestas son poco claras: ¿Cuándo podemos decir que concluyó la inserción laboral de un joven? Detrás de esta pregunta prevalece una concepción de la inserción laboral que se distingue de la que subyace al análisis basado en diversos indicadores del mercado de trabajo (tasas de actividad, de empleo, de desempleo); además, remite a un proceso social del cual pretende determinar los criterios que identifican el comienzo y el final.

Vernières (1997) define la inserción laboral como un proceso por el cual un individuo que, en otro tiempo, nunca perteneció a la población activa, logra una posición estabilizada en el sistema de empleo. Al respecto, el término "estabilizado" no remite necesariamente al hecho de que este individuo haya obtenido un empleo permanente a tiempo completo, sino al hecho de que su posición en el mercado laboral no evoluciona más y que, por ejemplo, empalmando una serie de empleos precarios, este permanecerá claramente prisionero de un segmento secundario o, peor aún, afectado por mucho tiempo por el desempleo, parecerá estar involucrado en un proceso de exclusión del mercado laboral. Teniendo en cuenta la complejidad de la noción de inserción, Vernières muestra que esta recubre diversas realidades, al encuentro por un lado, de demandas de trabajo que varían según los tipos de empleadores y, por otro lado, de categorías de mano de obra heterogéneas cuyas capacidades de acción varían en función de las características socio demográficas, escolares y laborales de los individuos que las componen.

Vincens (1997) se esforzó por enriquecer la definición económica y cuantitativa de la inserción utilizando el modelo del jobsearch de los economistas desde una perspectiva que podríamos calificar de "más sociológica" porque esta intentó escapar al paradigma del individuo que basa sus elecciones sobre la única racionalidad optimizadora. De este modo, esta perspectiva permitió a Trottier (2000) definir "la inserción [no solo como] el periodo durante el cual el individuo comienza a dedicar tiempo a la búsqueda de un trabajo con fines de lucro o al trabajo comercial en sí mismo, aun cuando antes, su tiempo se dividía entre el trabajo ad honorem, las actividades de ocio y los estudios" (p. 99), sino también la definía como la ejecución de un proyecto de vida que va mucho más allá de la obtención del primer trabajo. Por consiguiente, "el objetivo del análisis de la inserción no solo consiste en describir el periodo que separa la decisión de entrar en la vida activa y el primer empleo, sino el que se emplea hasta la realización del proyecto" (Trottier, Laforce y Cloutier, 1999, p. 312).

Otros trabajos, como los de Dubar (1995) o de Mauger(1998) también ampliaron la problemática de la inserción laboral perforando el horizonte del mercado de trabajo. Por consiguiente, la inserción es considerada como un paso progresivo hacia un estatus de trabajador provisto de responsabilidades productivas luego de un periodo de socialización. Por lo tanto, la inserción aparece como un periodo de 
adquisición de una identidad social y profesional, es decir, como una etapa del proceso de socialización de los jóvenes hacia la vida adulta. "La inserción, en esta perspectiva, escribe Mauger (1998), es el cierre de la juventud" (p. 55)

Inspirándose de la visión de Vernières (1997) y de Vincens (1997), pero también de los trabajos de Couppié y Mansuy (2001), Vultur y Trottier (2010) hacen de la inserción un proceso dinámico que caracteriza el paso de los jóvenes del sistema educativo a una posición relativamente estabilizada en el mercado laboral. Este proceso no se reduce a un periodo que empezará en el momento en que un joven comienza a buscar empleo y que terminará cuando lo encuentre; en efecto, este primer empleo puede ser provisorio y, tomando en cuenta los diversos modos de frecuentación de la escuela, puede combinarse con la continuación de los estudios ${ }^{8}$. La inserción laboral se desarrolla en un periodo en el que se mezclan situaciones de búsqueda de empleo, de desempleo, de formación y de inactividad. En esta perspectiva, este periodo no corresponde al mero momento que registra el acceso del joven a un empleo, sino que se inicia cuando este destina su tiempo a un empleo remunerado o a la búsqueda de trabajo en una mayor proporción que las actividades de ocio, los estudios y el trabajo no remunerado. La inserción termina cuando: a) el joven cesa de dedicar tiempo a la búsqueda de empleo o de estudios con el fin de acceder a otro trabajo, b) el joven ocupa un empleo permanente que puede ser el mismo en un futuro cercano y que no se aleje demasiado de sus expectativas, eventualmente reducidas tras experiencias adquiridas en el mercado laboral y c) el joven considera que debe mantener o aceptar este empleo, en función de los datos que posee sobre el mercado laboral. Por ende, este empleo le permite satisfacer sus necesidades, alcanzar una autonomía financiera y formar proyectos de vida viables. La estabilidad laboral, es decir el hecho de que el joven no prevea perder su empleo ni abandonarlo deliberadamente, y el hecho de que este empleo le otorgue una cierta autonomía y haga posible la realización de sus proyectos de vida, constituyen dimensiones centrales del proceso de inserción laboral. Esta concepción incluye a la vez criterios objetivos que permiten hacerse una idea de la conclusión (o no) del proceso y de los elementos subjetivos unidos a las representaciones que los jóvenes se deben hacer de su inserción. La atención se centra en la estabilidad laboral que no solo está relacionada con el hecho de contar con un contrato por tiempo indeterminado, sino también con la capacidad de un joven para mantenerse en el mercado laboral y no caer en el desempleo, aunque disponga de un empleo fijo. La expresión "posición estabilizada" de esta definición de inserción destaca el hecho de que un joven puede perfectamente ocupar de forma permanente posiciones inestables sobre todo si el funcionamiento del mercado laboral le impone ocupar con el tiempo empleos precarios.

Pese a estos avances, en función de estos breves panoramas se demuestra que la definición de la noción de inserción sigue siendo vaga, ya que no está provista de una definición unánimemente reconocida. "El mismo concepto de inserción es frecuentemente utilizado sin que se sepa realmente lo que abarca, debido a la falta de definición o al contrario, de una pluralidad de definiciones implícitamente admitidas" (Giret, 2000, p. 13). Por otra parte, estas tentativas de medición y de definición de la inserción laboral llevan a concluir, por el momento, que resulta imposible conseguir que converjan estos diversos enfoques. En efecto, unos y otros ocultan numerosas zonas de incertidumbre pese a los esfuerzos de las partes interesadas. "Se tiene incluso la sensación de que varios problemas, que solo fueron abordados recientemente, podrían ser encarados de manera diferente, y que incluso datos recopilados en el transcurso de trabajos ya efectuados podrían ser interpretados de forma diferente si se dispusiera de mejores herramientas conceptuales" (Trottier, 2000, p. 101). Bonniel y Lahire(1994) incluso afirman que "la noción de inserción es una categoría de la práctica y no un concepto (sea calificado de empírico o de teórico) a partir del cual el análisis sociológico podría operar" (p. 22). En su opinión, el verdadero objeto de estudio del sociólogo debería estar constituido "de las diferentes utilizaciones del término por agentes que producen discursos a partir de diversos lugares institucionales (organismos de formación, organismos públicos de investigación, de estadísticas, ministerios, trabajadores sociales, etc.)" (p. 29). En este aspecto, así como ya lo propuso el enfoque de las fuentes 
estadísticas quebequenses y francesas, la consideración de las especificidades sociales podría constituir una etapa ineludible de todo proceso que pretende integrar las diferentes dimensiones de la inserción laboral.

\section{4. ¿UN ANÁLISIS SOCIETAL DE LA INSERCIÓN?}

En el panorama institucional y académico diversificado, que abarca la inserción de los jóvenes, se desarrolló un enfoque inspirado en el análisis societal elaborado a fines de los '70 por Maurice, Sellier y Silvestre (1982), con motivo de una comparación en profundidad entre Alemania y Francia, que permite detectar coherencias estructurales propias a cada país. La socialización de los jóvenes y la construcción de sus cualificaciones, constitutivas de un "rapport educativo", es el centro de su análisis a condición de que se comprenda su interacción con el "rapport industrial" (las relaciones laborales) y el "rapport organizacional” (la división y la organización del trabajo).

\subsection{Un marco de análisis: configuraciones de actores, mediaciones sociales y experiencias individuales}

Este marco de análisis de la inserción laboral de los jóvenes será precisado por Silvestre (1987). Este autor se diferencia especialmente de los análisis de la inserción que predominan y la conciben como un mecanismo de circulación de cohortes hacia el mercado laboral, planteando la "prioridad cronológica del acto educativo con respecto al acto productivo". Al contrario, resalta la necesidad de construir una "causalidad circular" entre la construcción de los empleos y de las cualificaciones, por un lado, y la estructuración del sistema educativo, por el otro. Es en esta perspectiva que se va a situar Verdier (1996) quien se esfuerza, además, en calificar los cambios societales que afectan la inserción de los jóvenes franceses ${ }^{9}$; muestra que las nuevas generaciones están claramente más capacitadas que sus mayores, particularmente gracias al importante desarrollo de la formación profesional, y que ese compromiso entre una regulación académica de la educación —siempre predominante- y el surgimiento de reglas neo corporativistas en materia de formación, expone a los jóvenes a un mecanismo de exclusión selectiva (Garonna y Ryan, 1989) que prevalece en el mercado laboral francés, en detrimento de los que no tienen títulos y de los que obtuvieron los primeros niveles de certificación, aun cuando estos últimos sean, formalmente, objeto de un reconocimiento social. Desarrollando un enfoque similar, Lefresne $(2003,2010)$ analiza los "sistemas nacionales de inserción laboral" como "el conjunto de las normas y de las instituciones que supervisan el acceso de los jóvenes a la relación salarial” (p. 12) y, en este sentido, conducen a analizar "un modo de articulación formación/empleo" como un componente del "conjunto de los procesos de movilidad de la mano de obra". Del mismo modo, Walther (2006) define los regímenes de transiciones (transition regimes) según las experiencias biográficas de los jóvenes introducidos en diferentes contextos nacionales ${ }^{10}$; se inscribe así en una perspectiva ya iniciada por Rose (1984). Constatando que las dificultades crecientes de inserción suscitan intervenciones cada vez más recurrentes y consecuentes de los poderes públicos, él propone considerar este proceso como una "transición laboral". Esta última toma diversas modalidades que son permanentemente modificadas por los cambios de los dispositivos institucionales así como por los rapports de poder entre los jóvenes, el Estado, las empresas y los intermediarios del mercado laboral. En este marco, "la transición en el mercado laboral es considerada desde una lógica societal que traduce configuraciones de actores específicos y también especificidades culturales de la juventud, irreductibles a simples determinaciones económicas"(Dubar, 2001, p. 30). Más allá de las lógicas universales, los caminos de los jóvenes en el mercado de trabajo se inscriben en contextos nacionales cuya estructuración y regulación difieren. Prolongando esta perspectiva e integrando la incorporación de la experiencia de los jóvenes, Van de Velde (2008) destaca que: en Francia, clásicamente buscan "posicionarse" gracias al fortalecimiento de la profesionalización por medio del estudio; en el Reino-Unido se proponen "ser 
independientes" aunque tengan que dejar de estudiar precozmente para conquistar su autonomía; en España buscan "integrarse" con la ayuda de la familia para terminar por acceder (tardíamente) a una vivienda propia; finalmente, en Dinamarca tratan de “encontrarse a sí mismos” luego de sucesivos tanteos sostenidos a lo largo del tiempo por el sistema de protección social. En cuanto a esto, ¿qué análisis se puede realizar en una perspectiva societal del tema de la inserción de los jóvenes en el caso de Francia y de Quebec?

\subsection{Factores estructurales y contextuales que moldean la inserción de los jóvenes y sus modos de análisis en Francia y en Quebec}

Como vimos, el tema de la inserción laboral de los jóvenes toma lugar y sentido en los espacios nacionales que presentan grados y tipos de exigencia muy diferentes. Esta situación no es ajena a las especificidades de los "regímenes de Estado bienestar" (Esping-Andersen, 1999) que se desplegaron en Francia y en Quebec. El modelo "corporativista-continental" francés dio lugar a un sistema de relaciones contractuales relativamente cerrado en el cual empresarios, representantes del Estado y sindicatos se reúnen o se reunían regularmente para negociar salarios, prestaciones sociales o diversas cuestiones, como la formación profesional que es objeto, regularmente, de "leyes negociadas ${ }^{11}$ ". En Quebec, una forma atenuada de Estado bienestar (que podemos llamar "liberal-residual") no regula tanto el mercado laboral y favorece un sistema de empleo "de relaciones abiertas". En este aspecto, Francia se caracteriza por un mercado laboral más protegido, que asegura ciertas garantías y ciertos derechos a los empleados, mientras que Quebec se distingue por un mercado laboral más flexible y desregulado que pone a los principiantes y a los activos experimentados en competencia generalizada. Es así como, por ejemplo, en Quebec, al contrario de Francia, el empleo atípico, en general, no goza de un control jurídico específico. Además, el trabajo temporal, incluida la actividad de las agencias de empleo, no está en absoluto reglamentada en Quebec mientras que en Francia, el trabajo temporal está sujeto a restricciones jurídicas (Vultur y Bernier, 2014).

Los diferentes regímenes de Estado bienestar hacen referencia a concepciones específicas de las políticas públicas de empleo con respecto a los jóvenes. Si, en los dos casos, estas políticas supuestamente facilitan la entrada a la vida activa, dos modelos de dispositivos de inserción se encuentran en acción: dicho sintéticamente, se trata de un "modelo integrado" francés y de un "modelo dividido" quebequense. En ninguno de los dos casos, las articulaciones entre las políticas públicas de empleo y la inserción en el mercado laboral se reducen a relaciones mecánicas y establecidas; estas articulaciones son multiformes e intervienen en diversas etapas de las trayectorias de los jóvenes. Sin embargo, las políticas de empleo tienen un carácter mucho más puntual en Quebec mientras que en Francia representan alternativas más durables para el trabajo; en este país esas políticas se convirtieron en un verdadero componente estructural de la inserción, prioritariamente de jóvenes de 16 a 25 años con dificultares en el mercado laboral, lo que contribuye a la institucionalización de grupo etario (Verdier, 1995). En esta configuración en la que el Estado desempeña un rol tutelar, los jóvenes constituyen una categoría administrativa muy delimitada. En el modelo quebequense, la juventud constituye ante todo una "fase de transición" que debe concluir rápidamente gracias a un acceso precoz al mercado laboral. Por el hecho de favorecer fuertemente la flexibilidad laboral e impulsar la individualización de los riesgos, este tipo de Estado bienestar orienta a los jóvenes hacia transiciones profesionales más fragmentadas, más reversibles y más indeterminadas.

De esta manera, en cada sociedad, el proceso de inserción de los jóvenes se rige con reglas específicas relativas a las condiciones de participación en la formación y en el trabajo. Así, en Quebec, el sistema de enseñanza favorece una disposición muy individualizada de las trayectorias con una multiplicidad de programas y de planes de estudios y con posibilidades importantes de cambio de orientación o de articulación entre estudios y trabajo remunerado (Charbonneau, 2007; Eckert, 2009; Bourdon, Longo y Charbonneau, 2016). Así, los jóvenes ingresan más temprano a la vida activa, se encuentran más a menudo en situaciones de 
combinación de estudios/trabajo y tienen mayor facilidad de acceso a empleos, eventualmente transitorios. En Francia, los recorridos de formación se inscriben tradicionalmente en un modelo de transición escuela/ trabajo más lineal y exclusivo, que, por cierto, conduce a los dispositivos de investigación a minimizar la importancia del trabajo en periodo de estudios (Moulin, 2011) que, por lo tanto, tiende a expandirse bajo diversas influencias: el desarrollo de la alternancia (aprendizaje, contrat de professionnalisation ${ }^{12}$, pasantías) y de experiencias de trabajo que manifiestan a la vez la necesidad de financiar estudios que se prolongan, y la preocupación de "encontrarse a sí mismos" al concluir itinerarios personales (Van de Velde, 2008 ${ }^{13}$ ). Francia sigue privilegiando la importancia de los títulos obtenidos, con consecuencias tanto en las representaciones individuales como en las prácticas de reclutamiento de los empleadores ${ }^{14}$. En cambio, en Quebec prima la experiencia (Vultur, 2007); para aprobar su inserción laboral, los jóvenes quebequenses se apoyan en mayor medida en sus redes de contacto social y en sus experiencias de trabajo y se encuentran, por periodos más largos, en situaciones de "floundering", es decir en empleos muy diversos que a menudo tienen pocos vínculos con su formación (Gauthier, Hamel, Molgat, Trottier y Vultur, 2004; Vultur, 2016).

Como vimos, los instrumentos de que disponen Francia y Quebec para observar los recorridos de inserción laboral, no se basan en las mismas categorizaciones estadísticas. En Francia, los dos tiempos sociales —estudio y vida activa - aparecen como casilleros estancos; mientras que en Quebec y en Canadá, prevalece una lógica combinada ${ }^{15}$. Entonces, "estar estudiando" no designa la misma realidad en las investigaciones longitudinales del CÉREQ de Francia y en aquellas sobre los jóvenes en transición (EJET) de Estadística de Canadá. Dentro de la categorización estadística francesa, cada tipo de situación es a priori exclusivo a diferencia de la categorización canadiense donde un joven puede estar estudiando y trabajando. Es evidentemente una información esencial para quien quiera "comparar" los índices de actividad de los jóvenes en Francia y en Quebec.

\section{LA INSERCIÓN LABORAL: ¿MADUREZ Y SUPERACIÓN DE UNA NOCIÓN?}

El marco del rapport salarial en el cual la noción de inserción laboral fue forjada emprendió una transformación de tal amplitud que las representaciones y las realidades producidas por esta noción deben cambiar. Ciertas dimensiones que caracterizan la situación de los jóvenes en el mercado laboral ya no pueden ser interpretadas en términos de inserción: nuevas nociones están en búsqueda de legitimación, como así lo demuestra el surgimiento o el resurgimiento de categorías tales como recorridos y transiciones. Significativamente imbricadas, estas parecen poder caracterizar más eficazmente los efectos de las recomposiciones de la educación y del mercado laboral en las trayectorias escolares y profesionales de los jóvenes. Una vez más, es necesario llegar a comprender en conjunto las categorizaciones que moviliza la acción pública, los conceptos en los cuales se apoyan las estadísticas públicas y las categorías de análisis producidos por los investigadores.

\subsection{El surgimiento de la noción de recorrido laboral como categoría de referencia}

Desarrollada en el campo político, inicialmente para comprender los lazos entre trayectoria de empleo, formación continua y desempleo, la noción de recorrido labora ${ }^{16}$ vio sus usos extenderse a la formación inicial para inscribirse en adelante en el marco más amplio de un enfoque transicional de los recorridos de formación y de empleo. Primero, la noción de recorrido laboral surgió y proliferó en los discursos políticos y en los dispositivos de acción pública en materia de empleo/capacitación. Esta noción resulta a la vez de una desestabilización de la relación salarial clásica basada en el empleo estable y de la búsqueda titubeante de una alternativa que consistiría en asegurar que los individuos puedan "protegerse individualmente contra los avatares y cultivar su 'capacidad de navegar' en los mercados laborales" (Mériaux, 2009, p. 11). En este 
contexto, varios factores contribuyen a legitimar la noción de recorrido (Collectif, 2014): su compatibilidad con una concepción potencialmente desestandarizada de la vida laboral; su aptitud para dar cuenta de las elecciones diversificadas de los individuos (a diferencia de la noción de trayectorias que supone una concepción lineal y determinista de las experiencias escolares y profesionales); su acepción pluralista de los dispositivos públicos, pensados no solo con respecto a su dimensión posiblemente restrictiva, sino también en función de las oportunidades ofrecidas a las personas para que desarrollen proyectos. Así, la noción de recorrido "informa acerca de las interacciones posibles entre las diferentes temporalidades constitutivas de una existencia y su asunción mediante la acción pública: vida profesional, vida familiar, vida social" (Collectif, p. 15), que permite remarcar la multiplicidad de factores que intervienen en la biografía de un individuo (Longo, 2016).

Más allá del "vasto movimiento de redefinición de las responsabilidades respectivas del empleado, de la empresa y de las instituciones públicas" (Mériaux, 2009, p. 12), la búsqueda de protecciones no estaría en adelante tan focalizada sobre las categorías socio profesionales como sobre las écurisation ${ }^{17}$ de los recorridos laborales de los individuos a lo largo de su ciclo de vida, al desarrollar la prevención más que la reparación, registro privilegiado por el Estado bienestar elaborado luego de la Segunda Guerra mundial. En este nuevo contexto, las temáticas de la empleabilidad y de la activación de las políticas sociales constituyen el núcleo del modelo de Estado social "posfordista" (Mercure y Vultur, 2010) que empezaría a tomar forma. Dos grandes concepciones políticas predominan, por un lado, los enfoques que pretenden "equipar a los individuos para el mercado" y, por el otro, los que tratan más bien de "equipar el mercado para los individuos"(Gautié, 2003).

Por lo tanto, el surgimiento de la categoría de recorrido dio lugar a la retórica de la "sécurisation de los recorridos laborales" que debió conciliar visiones antagónicas del funcionamiento del mercado laboral y promover un equilibrio virtuoso entre responsabilización de los individuos y construcción de nuevas garantías colectivas. En Francia, este surgimiento apareció primero en dispositivos puestos en marcha por la negociación colectiva antes de "imponerse como un nuevo referencial de las políticas de empleo/ capacitación” (Mériaux, 2009, p. 13) ${ }^{18}$. En este marco, la individualización es una característica central para las diversas dimensiones de los recorridos que se presentan en el plano pedagógico con modalidades personalizadas de aprendizaje, en el plano estatutario con la búsqueda de una continuidad jurídica entre las diferentes etapas de la carrera personal, y en el plano psicosocial con la construcción de un proyecto laboral motivador y realista. Pero, además de la retórica que efectivamente impregnó de manera amplia la acción pública, las prácticas tienen dificultades para combatir la fuerte selectividad del mercado laboral, por falta de una instrumentación adecuada. Por otro lado, los trabajos empíricos muestran que la sécurisation está significativamente ajustada a la capacidad de los actores y de los instrumentos de articular el desarrollo de las competencias individuales ya una gestión preventiva de los riesgos del empleo. En Quebec, el tema de la sécurisation comienza apenas a desarrollarse en el debate público, sabiendo que el contexto norteamericano del mercado del trabajo dificulta su legitimación y su operacionalización ${ }^{19}$.

\subsection{La actualidad de la noción de transición laboral}

El enfoque en términos de "mercados transicionales de trabajo" propuesto por economistas heterodoxos (Gazier, 2003) recomienda el emplazamiento de dispositivos destinados a "proteger" los recorridos laborales cargando la responsabilidad de los riesgos del empleo en las instituciones colectivas, por ejemplo, por la mediación de contratos de transición entre dos empleos, que articulan capacitación y trabajo de jornada parcial. Las mediaciones institucionales entre los individuos y los avatares económicos y sociales pueden ser diversificadas, asociando actores públicos y privados, sectoriales o interprofesionales, a diferentes escalas. Estas mediaciones no pretenden garantizar el acceso a situaciones estables, sino más bien favorecer variaciones construidas con el fin de evitar el establecimiento de un precariado estructural. Esta atención de los 
riesgos que afectan a los recorridos laborales debe tener como mira una ciudadanía social renovada que articularía responsabilidad individual y responsabilidad colectiva, siendo esta última predominante. La misma denominación de "mercados transicionales" muestra que los recorridos de inserción son concebidas como variaciones o transiciones, entre otras, pero cuyas modalidades de sécurisation variarán según las características de los individuos, de las empresas y de las modalidades preexistentes de acompañamiento hacia el empleo y la calificación. Los estudios recientes de la administración experta a la francesa ${ }^{20}$ se inscriben en esta perspectiva muy general, destinada a comprender el conjunto de las transiciones en el mercado laboral, incluidas las de los jóvenes; de esta manera vuelve al sendero trazado hace cuarenta años en el marco de la Commissariat Général du Plan. No es trivial notar que las ayudas más emblemáticas a la inserción laboral de los jóvenes, tales como los contratos de aprendizaje y de profesionalización, han dejado de estar jurídicamente reservados a los menores de 26 años.

La teoría de las capacidades (Sen, 1999) ha tenido desde hace una década un resultado considerable en los análisis de los recorridos de capacitación y de empleo y por lo tanto de los procesos de inserción. Recordemos que la noción de capacidad (o capabilite $e^{21}$ ) refiere al "poder de actuar" cuyas dimensiones constitutivas son la libertad de elegir, el poder de acompañamiento y la responsabilidad colectiva (Bonvin y Farvaque, 2007). Como en el caso de los "mercados transicionales", la inserción se convierte en un paso entre el conjunto de las transiciones a considerar, sabiendo que necesita explícitamente integrar los recorridos de formación inicial con el fin de estar en condiciones de considerar la constitución de las capacidades de las personas, principalmente con respecto a las posibilidades de elección ofrecidas a los individuos, sobre todo durante los procesos de orientación: se trata así de determinar si las orientaciones y las transiciones que resultan de este proceso son elegidas o padecidas. Por tanto, una de las metas metodológicas será saber si es posible juntar los datos, especialmente en el momento de las encuestas o de las entrevistas, con el fin de tomar "plenamente en consideración las expectativas y la subjetividad de las personas [...], comparando estos resultados con datos factuales" (Bonvin y Farvaque, 2007, p. 16-17). Recientemente, Leclercq (2014) se focaliza en los recorridos laborales de encargados de "higiene, calidad, seguridad, medio ambiente" que él aprehende como procesos sociales. Con respecto a esto, el autor toma la noción de "recorridos de transición" con el fin de considerar todas las formas de continuidad y de ruptura a las cuales esos empleados pueden enfrentarse. De esta manera, referirse a las transiciones permite analizar el "debilitamiento" o, al contrario, la "sécurisation", o incluso "la innovación” que caracteriza la construcción de los recorridos (Leclercq, 2014, p. 49).

En resumen, cualquiera que sea el paradigma de referencia, como lo constataba Rose (1996), la noción de transición laboral

puede ser un excelente prisma para el análisis de la transformación en proceso de relaciones de trabajo y de empleo, el rol creciente de las empresas en la capacitación, el desacoplamiento entre la actividad profesional y los recursos de los individuos, la precariedad creciente de las categorías socio profesionales y de los contratos, el rol esencial de las instancias de regulación estatal o intermedias, [...] la interpenetración de las actividades de trabajo y de las actividades extra laborales etc., el dualismo reforzado... Todos estos fenómenos observados en la transición laboral son quizá anticipaciones de nuevas relaciones de trabajo y de empleo, características del surgimiento en proceso de un nuevo asalariado. (p.8)

\subsection{Recorridos laborales, recorridos de formación y transición: entre categorías políticas y categorías académicas}

Independientemente del enfoque adoptado, los análisis son necesariamente dependientes de la disponibilidad de fuentes estadísticas idóneas. Si volvemos a las transiciones entre la escuela y el empleo, se trata de saber si las fuentes estadísticas están en condiciones de producir los datos necesarios para la caracterización de los recorridos. Desde hace quince años, la presentación oficial de los resultados de las encuestas Génération del CÉREQ institucionalizó en Francia tipologías de recorridos que sacan provecho de los datos incluidos en los calendarios mensuales que registra esta fuente. A partir de análisis factoriales, 
construye una clasificación de recorridos de tres años en la que distingue un "acceso durable en el empleo", una "sécurisation diferida", un "desenganche temporal del empleo", un "cambio fuera del empleo", "recorridos marcados por el desempleo y la inactividad” y "regresos a la formación”. Esta presentación se aleja de las aporías de las evaluaciones estáticas de la inserción con el fin de calificar los recorridos, sabiendo que, aunque solo sea a título parcial, los indicadores que fundan tales tipologías tienen en cuenta la manera en que la oferta de empleo se dirige a los jóvenes (Epiphane, Giret, Hallier, Lopez y Sigot, 2001).

Además de estos ejercicios tipológicos, la transformación de algunas encuestas Génération en estudios de panel, gracias a las interrogaciones que conforman las muestras que se les hace a los individuos tres años después de su salida del sistema educativo, abre nuevas perspectivas analíticas. Así la generación de 1998 fue interrogada en cuatro ocasiones para cubrir un horizonte de diez años y abordar así más a fondo las dimensiones procesuales de la inserción. Con respecto a esto, "un horizonte de diez años se concibe como un término en el cual una cohorte está ya introducida en el sentido en que los valores de los índices de empleo y de desempleo que la caracterizan alcanzaron los valores observados en el conjunto de la población activa" (Dupray, 2012, p. 10, refiriéndose a Vincens, 1997). De este modo, reuniendo recorridos de más larga duración, Géhin y Palheta (2012) están en posición de examinarlas condiciones que hacen posible una integración durable de los jóvenes no calificados en el mercado laboral y de volver a lanzar un análisis que se refiera a la integración, es decir a la consideración de muchas otras dimensiones y no solo a la única inserción en el empleo.

Solo queda que dichas fuentes, fijadas en "edades de inserción", se reconozcan menos eficaces para inscribirse plenamente en un enfoque cuantificado de las transiciones, particularmente porque estas solo integran de una manera limitativa los recorridos académicos y las diferentes transiciones e interacciones a las cuales dan lugar. Fuentes como la EJET, que contienen los datos de panel de quienes se sigue a partir de los 15 años, resultan claramente más adecuadas. Es también el caso del panel suizo TREE (transición escuela empleo) realizado a partir de las muestras de jóvenes de 15 años entrevistados en el marco de la encuesta PISA de la OCDE. Cabe añadir que desde hace tiempo, el lugar de la formación en alternancia de los jóvenes, por un lado, y de los análisis en términos de transición, por el otro, están estrechamente vinculados. En 1995, Jobert, Marry y Tanguy escribían:

A diferencia de Gran Bretaña y sobre todo de Francia, las investigaciones en Alemania substituyen a la noción de inserción laboral, por la de transición, que conjugan en plural [...]. A partir de investigaciones longitudinales, estas tratan de desenmarañar los efectos de edad (de ciclo de vida), de periodo (o de coyuntura económica) y de generación. (p. 17)

En Alemania como en Suiza, es usual analizar la "doble transición”: entre la escuela obligatoria y el aprendizaje; entre el aprendizaje y el empleo ordinario. Además, los recientes trabajos comparativos muestran la necesidad de integrar plenamente en estos enfoques societales los factores simbólicos y subjetivos que contribuyen a moldear de manera individualizada, más allá de los orígenes sociales y escolares, las relaciones que los jóvenes mantienen con la vida profesional: estos factores constituyen dimensiones claves de "la construcción de los recorridos [...], esto no solamente a escala biográfica [...] sino igualmente a nivel de las especificidades sociales" (Longo, 2014, p.87), destacando que así como "la caducidad de la idea según la cual la sociedad sería un todo coherente, un sistema que determina las prácticas y las representaciones de los individuos, desplaza el interés de la investigación hacia los actores, sus compromisos y sus construcciones de la realidad que los rodea [...]" (Longo, p. 86).

\section{Conclusión}

El tema de la inserción laboral de los jóvenes apareció en un contexto histórico muy preciso. Proviene de la matriz del Estado social a la francesa orientada, inicialmente, a favorecer el acceso al empleo de las personas con discapacidad. Socialmente construido, el concepto de inserción laboral está relacionado con las políticas 
públicas que supuestamente harían más eficaz la participación de los jóvenes en el mercado laboral. Estas políticas, que necesitan una producción de conocimientos sobre la articulación de la formación y de los empleos, suscitaron la aparición de organismos de estudios, de estadisticas y de investigación que definieron y operación alizaron el concepto de inserción laboral. Esta doble dinámica, institucional y cognitiva, es componente del ADN del concepto de inserción laboral.

Originalmente, los estudios sobre la inserción laboral fueron concebidos para responder a las necesidades de planificación de las instancias gubernamentales. Primero, la inserción fue relacionada con los problemas de los jóvenes en dificultad. Más en general, los trabajos se han esforzado comprender la diversidad creciente de los desarrollos de los jóvenes en el mercado laboral. Se han multiplicado, pero no se ha desarrollado una teorización general del objeto de investigación, sobre todo porque cada investigador desarrolló su propio enfoque de la inserción en función de preguntas específicas. Aún falta que los análisis comparativos denominados societales identifiquen coherencias en las formas de categorización, que estén sujetos a la producción estadística, a la acción pública o a los análisis "académicos".

Hoy en día, la noción de inserción laboral se hace más imprecisa que nunca, sobre todo porque está destinada a expandirse para dar cuenta de los procesos cada vez más diversificados que tienden a integrar las fases relativas a los estudios como las que se refieren a las carreras individuales. Su legitimidad científica siempre está en cuestionamiento y reconsideración, dentro de un contexto donde los "mundos" de la educación/formación como del trabajo están comprometidos en profundas transformaciones, en lugar de diferencias cada vez más marcadas, por un lado, entre el funcionamiento estructural de las instituciones de formación y del mercado laboral y, por el otro, los recorridos biográficas y los ciclos de vida de los individuos.

En tal contexto, las nociones de "recorridos" y de "transiciónes" aparecen heurísticas. Por consiguiente, los programas de investigación son llamados a reunir análisis y encuestas estadísticas longitudinales con recorridos de formación y recorridos laborales, pero también estudios cualitativos que analicen las interacciones entre lo estructural y lo biográfico al igual que comparaciones internacionales que permitan comprender el grado de coherencia entre las diversas formas de categorizaciones, políticas, estadísticas y experimentadas, que reúnan así las perspectivas comparativas indicadas por Ryan (2001). El estudio de los recorridos y de las transiciones darán todo su potencial a través de enfoques interdisciplinarios y de métodos mixtos (mixed methods), referentes a un análisis crítico de estas consideraciones oficiales.

Para finalizar, cabe señalar que los análisis presentados en nuestro artículo tienen, sin lugar a dudas, límites que se deben principalmente a la imposibilidad de considerar la totalidad de los trabajos realizados sobre el tema desarrollado. Con respecto a esto, no quisimos reunir demasiadas referencias bibliográficas, por tal motivo, apuntamos más que todo al uso de publicaciones que estén más relacionadas con los objetivos de nuestro artículo. En efecto, aún conscientes de la imposibilidad de hacer justicia a todos los trabajos pertinentes, privilegiamos la citación de los artículos sobre la inserción laboral de los jóvenes que tienen un carácter conceptual, producidos principalmente por investigadores de Francia y de Quebec. Ante la cantidad impresionante de investigaciones y de informaciones sobre el tema de la inserción laboral de los jóvenes y considerando los límites en términos de espacio del artículo, debimos ser selectivos, tratando de proponer un balance de los progresos y de las pistas de reflexión sobre una necesaria reconceptualización de la inserción laboral de los jóvenes.

\section{BiBliografía}

Barbier, J. - C. (2002). Peut-on parler d'« activation » de la protection sociale en Europe ? Revue française de sociologie, 43 (2), $307-332$.

Bonniel, J. et Lahire, B. (1994). Remarques sociologiques sur la notion d'insertion. In Guth, S. (dir.), L'insertion professionnelle (p. 21 - 31). Paris : L’Harmattan. 
Bonvin, J.-M. et Farvaque, N. (2007). L'accès à l'emploi au prisme des capabilités, enjeux théoriques et méthodologiques. Formation Emploi, 98, 9-24.

Bordigoni, M. et Vergnies, J. - F. (1997). Étudiants salariés. Quand la vie active commence avant la fin des études. Céreq Bref, 132 (juin).

Bordigoni, M., Demazière, D. et Mansuy, M. (1994). L'insertion professionnelle à l'épreuve de la jeunesse. Points de vue sur les recherches françaises. Communication au « Network on Transition in Youth », Seelisberb.

Bouffartigue, P., Lagrée, J. - C. et Rose, J. (1989). Jeunes : de l'emploi aux modes de vie. Points de vue sur un champ de recherche. Formation Emploi, 26, 63-75.

Bourdon, S. et Cleaver, A. (2000). Le genre et le stéréotype du diplôme lors de l'insertion professionnelle. Revue des sciences de l'éducation, XVII (3), 677-700.

Bourdon, S., Longo, M. E. et Charbonneau, J. (2016). Les figures de la réussite, des clés pour appréhender les bifurcations scolaires et professionnelles. Formation-emploi, 134, 27-45.

Castel, R. (1995). Les métamorphoses de la question sociale. Paris : Fayard.

Centre d'études et de recherches sur les qualifications (CÉREQ) (2014). Quand l'école est finie. Premiers pas dans la vie active de la génération 2010 - Enquête 2013. Marseille : CÉREQ.

Charbonneau, J. (2007). L'influence du contexte sociétal sur les trajectoires scolaires et professionnelles des jeunes adultes. In Bourdon, S. et Vultur, M. (dir.), Les jeunes et le travail (p. 53 - 70). Québec, Les Éditions de l'Institut québécois de recherche sur la culture (IQRC).

Charles, N. (2015). Enseignement supérieur et justice sociale. Sociologie des expériences étudiantes en Europe. Paris : La Documentation française. Collection «Études et recherches ».

Collectif (2014). Les dispositifs publics en action. Vers une sécurisation des parcours professionnels et personnels? Paris : Éditions Liaisons, Centre d'étude de l'emploi.

Coulon, A. (2012). L'École de Chicago. Paris : Presses universitaires de France. Collection « Que sais-je ? ».

Couppié, T. et Mansuy, M. (2001). Construction et usage des catégories d'analyse. L'insertion professionnelle des jeunes : éléments de comparaison européenne. 8e Journées d'études Céreq. Marseille : Lasmas-IdL.

Demazière, D. et Dubar, C. (1993). L'insertion professionnelle des jeunes de bas niveau scolaire. Marseille : CÉREQ. Collection « Documents ».

Desrosières, A. (2003). Historiciser l'action publique : l'État, le marché et les statistiques. In Laborier, P. et Trom, D. (dir.). Historicités de l'action publique (p. 207 - 221). Paris : Presses universitaires de France.

Desrosières, A. (2008). L'argument statistique. Paris : Mines ParisTech, Presses de l'école des Mines.

Dubar, C. (1995). La socialisation. Construction des identités sociale et professionnelles. Paris: Armand Colin.

Dubar, C. (1998). Réflexions sociologiques sur la notion d'insertion. In Charlot, B. et Glasman, D. (dir.), Les jeunes, l'insertion, l'emploi (p. 29-37). Paris : Presses universitaires de France.

Dubar, C. (2001). La construction sociale de l'insertion professionnelle. Éducation et sociétés, 7, 23-36.

Dupray, A. (2012). 10 ans de parcours professionnels des jeunes : l'intérêt des études

Eckert, H. (2009). Étudier, travailler... Les jeunes entre désir d'autonomie et contrainte sociale. Sociologie et sociétés, XLI (1), 239-262.

Eme, B. (2012). Insertion. In Bevort, A., Jobert, A., Lallement, M. et Mias, A. (dir.), Dictionnaire du travail (p. 401 407). Paris : Presses universitaires de France. Collection « Quadrige, DicosPoche ».

Epiphane, D., Gire t, J. - F., Hallier, P., Lopez, A. et Sigot, J.-C. (2001). GÉNÉRATION 1998 : à qui profite l'embellie ? Céreq Bref, 181 (décembre).

Esping - Andersen, G. (1999). Les trois mondes de l'État-providence: essai sur le capitalisme moderne. Paris : Presses universitaires de France. Collection « Le lien social ».

Flamand, J. (2016). Les transitions professionnelles, révélatrices d'un marché du travail à deux vitesses. La note d'analyse, 50, France Stratégie. www.strategie.gouv.fr. 
Garonna, P. et Ryan, P. (1989). Le travail des jeunes, les relations professionnelles et les politiques sociales dans les économies avancées. Formation Emploi, 25, 78-90.

Gauthier, M. (2001). La recherche sur les jeunes au Canada. In Gauthier, M. et Pacom, D. (dir.), La recherche sur les jeunes et la sociologie au Canada (p. 11-21). Québec : Les Éditions de l'Institut québécois de recherche sur la culture (IQRC).

Gauthier, M. (2007). De jeunes chômeurs à jeunes travailleurs : évolution de la recherche sur les jeunes et le travail au Québec depuis 1980. In Bourdon, S. et Vultur, M. (dir.), Les jeunes et le travail (p. 23-50). Québec : Les Éditions de l'Institut québécois de recherche sur la culture ('IQRC).

Gauthier, M., Hamel, J., Molgat, M., Trottier, C. et Vultur, M. (2004). L'insertion professionnelle et le rapport au travail des jeunes qui ont interrompu leurs études secondaires et collégiales. Étude rétrospective. Montréal : INRS Urbanisation Culture et Société.

Gautié, J. (2003). Marché du travail et protection sociale : quelles voies pour l'après - fordisme ? Esprit, novembre, $78-115$.

Gazier, B. (2003). Tous sublimes : pour un nouveau plein emploi. Paris : Flammarion.

Géhin, J. - P. et Palheta, U. (2012). Les devenirs socio-professionnels des sortants sans diplôme; un état des lieux dix ans après la sortie du système éducatif (1998-2008). Formation Emploi, 118, 15-36.

Giret, J. - F. (2000). Pour une économie de l'insertion professionnelle des jeunes. Paris : CNRS Éditions.

Guitton, C. (1998). Travail et ordre social. Une étude historique et juridique des politiques d'insertion par le travail. Travail et Emploi, 77, 15-38.

Hamel, J. (2007). Le rapport au travail et la génération numérique. In Bourdon, S. et Vultur, M. (d ir.), Les jeunes et le travail (p. 69-89). Québec: Les Éditions de l'Institut québécois de recherche sur la culture (IQRC).

Iribarne, A. (1986). L'insertion des jeunes dans la vie active : des problématiques qui restent à construire. In Tanguy, L. (dir.), L'introuvable relation formation-emploi. Paris : La Documentation française.

Jobert, A., Marry, C. et Tanguy, L. (1995). Mise en perspective d'un domaine de recherche en Allemagne, GrandeBretagne et Italie. In Jobert, A., Marry, C. et Tanguy, L. (dir.), Éducation et travail en Grande-Bretagne, Allemagne et Italie (p. 9-22). Paris : Bibliothèque des Sciences de l'Éducation, Armand Colin.

Jobert, B. et Muller, P. (1987). L'État en action. Politiques publiques et corporatismes. Paris : Presses universitaires de France.

Leclercq, E. (2014). Lorsque l'activité professionnelle recompose les parcours de transition : les responsables « Hygiène, qualité, sécurité, environnement ». Formation Emploi, 128, 47-64.

Lefresne, F. (2003). Les jeunes et l'emploi. Paris : La Découverte. Collection « Repères ».

Lefresne, F. (2010). Effets générationnels et transformations structurelles de l'emploi. Regards croisés sur l'économie, 7, 91-100.

Lichtenberger, Y. (1995). Alternance en France et qualification professionnelle. In Lichtenberger, Y., Luttringer J.-M. et Poupard, R. (dir), Alternance et formation professionnelle: les enjeux. Paris : Les Éditions d'Organisation.

Lochet, J.-F. (dir.) (2003). Entreprises et jeunes débutants. Paris: L'Harmattan. Collection « Dynamiques d'entreprises $\gg$.

Longo, M. E. (2014). Co-construire l'insertion professionnelle des jeunes. In Collectif de chercheurs de 7 laboratoires, Les dispositifs publics en action. Vers une sécurisation des parcours professionnels et personnels ? (p. 81-99). Rueil Malmaison : Liaisons Éditions.

Longo, M. E. (2016). Les parcours de vie des jeunes comme des processus. Les Cahiers dynamiques, 67, 48-58.

Massardier, G. (1996). Expertise et aménagement du territoire. L'État savant. Paris : L'Harmattan. Collection « Logiques politiques ».

Mauger, G. (1998). Jeunesse, insertion et condition juvénile. In Charlot, B. et Glasman, D. (dir.), Lesjeunes, l'insertion, l'emploi (p. 55-59). Paris : Presses universitaires de France.

Mauger, G. (2001). Les politiques d'insertion. Une contribution paradoxale à la déstabilisation du marché du travail. Actes de la recherche en sciences sociales, 136-137, 5-14. 
Maurice, M., Sellier, F. et Silvestre, J. - J. (1982). Politiques de l'éducation et organisation industrielle en France et en Allemagne : essai d'analyse sociétale. Paris: Presses universitaires de France. Collection « Sociologies 》.

Méhaut, P. (2006). Une scène nationale classique en voie de recomposition ? L'accord interprofessionnel et les accords de branche sur la «Formation tout au long de la vie ». In Mériaux, O. et Verdier, E. (dir.), Les relations professionnelles et l'action publique face aux risques du travail et de l'emploi (p. 25-46). Rapport pour le Commissariat général du Plan et la DARES, miméo LEST, Aix en Provence.

Mercure D. et Vultur, M. (2010). La signification du travail. Nouveau modèle productif et ethos du travail au Québec. Québec : Presses de l'Université Laval.

Mériaux, O. (2009). Les parcours professionnels : définition, cadre et perspectives. Éducation permanente, 181, 11-21.

Moulin, S. (2011). Enquêtes de jeunesses : une comparaison France - Canada des dispositifs statistiques nationaux. In Degenne, A., Marry, C. et Moulin, S. (dir.), Les catégories sociales et leurs frontières (p. 61-83). Québec : Les Presses de l'Université Laval. Collection « Société et Population ».

Moulin, S. et Doray, P. (2007). Le parcours des jeunes : catégorisations statistiques et représentations normatives. Communication présentée au colloque Approches longitudinales : confrontations franco-canadiennes. Paris, octobre.

Nicole - Drancourt, C. et Roulleau-Berger, L. (1995). L'insertion professionnelle des jeunes. Paris : Presses universitaires de France. Collection « Que-sais-je ? ».

Noblet, P. (2014). Les enfants de Don Quichotte. De la «stabilisation » au « logement d'abord ». Le sociographe. Recherches en travail social, 48, 11-20.

Paul, J.-J. (1989). La relation formation-emploi: un défi pour l'économie. Paris : Economica. Collection « Économie contemporaine ».

Pottier, F. (1990). La difficile insertion professionnelle des jeunes. CÉREQ BREF, 58.

Rioux, M. (1969). Jeunesse et société contemporaine. Montréal : Presses de l'Université de Montréal.

Rose, J. (1984). En quête d'emploi. Paris : Economica.

Rose, J. (1996). L'organisation des transitions professionnelles entre socialisation, mobilisation et recomposition des rapports de travail et d'emploi. Sociologie du travail,1, 63-79.

Ryan, P. (2001). The School-to- Work Transition : A Cross-National Perspective. Journal of Economic Literature, 39 (1), 34-92.

Saint - Pierre, C. (2001). L'insertion professionnelle et citoyenne des jeunes au Québec. In Roulleau - Berger, L. et Gauthier, M. (dir.), Lesjeunes et l'emploi dans les villes d'Europe et d'Amérique du Nord (p. 57-72). Paris : Éditions de l'Aube.

Sarfati, F. (2014). Professionnalisation des études et désir d'insertion professionnelle. In Collectif de chercheurs de 7 laboratoires, Les dispositifs publics en action. Vers une sécurisation des parcours professionnels et personnels? (p. 111-129). Rueil-Malmaison : Éditions Liaisons.

Schwartz, B. (1981). L'insertion des jeunes en difficulté. Paris : La Documentation française.

Sen, A. (1999). Éthique et économie. Paris : Presses universitaires de France.

Silvestre, J. - J. (1987). Éducation et économie: éléments pour une approche sociétale. Aix-en-Provence : LEST - CNRS, Mimeo.

Tanguy, L. (dir.) (1986). L'introuvable relation formation-emploi. Paris : La Documentation française.

Tanguy, L. (2002). La mise en équivalence de la formation avec l'emploi dans les IV e et V e Plans (1962 - 1970). Revue française de sociologie, 43 (42), 685-709.

Trottier, C. (2000). Questionnement sur l'insertion professionnelle des jeunes. Lien social et Politiques, 43, 93-101.

Trottier, C., Laforce, L. et Cloutier, R. (1999). Les représentations de l'insertion professionnelle chez les diplômés de l'université. In Charlot, B. et Glasman, D. (dir.), Les jeunes, l'insertion, l'emploi (p. 310-341). Paris : Presses universitaires de France.

Van de Velde, C. (2008). Devenir adulte en Europe : sociologie comparée de la jeunesse en Europe. Paris : Presses universitaires de France. Collection « Le lien social ». 
Verdier, E. (1995). Politiques de formation des jeunes et marché du travail, la France des années 80. Formation Emploi, 50, $19-40$.

Verdier, E. (1996). L'insertion des jeunes « à la française » : vers un ajustement structurel ? Travail et Emploi, 69, 37-54.

Vernières, M. (1997). L'insertion professionnelle. Analyses et Débats. Paris : Economica.

Vincens, J. (1997). L'insertion professionnelle des jeunes. À la recherche d'une définition conventionnelle. Formation Emploi, 60, 21-36.

Vultur, M. (2007). Les critères de sélection de la main-d'œuvre et le jugement sur les compétences des candidats à l'embauche au Canada : quelques éléments d'analyse. Cabiers de recherche sur l'éducation et les savoirs, 6, 235-254.

Vultur, M. (2016). Les stratégies professionnelles des jeunes Québécois, salaries d'agence de travail temporaire. In Meilland, C. et Sarfati, F. (dir.), Accompagner vers l'emploi.Quand les dispositifs se mettent en action (p. 225-238). Paris : Éditions Peter Lang.

Vultur, M. et Bernier, J. (2014). Les agences de travail temporaire : leur rôle et leur fonctionnement comme intermédiaires du marché du travail. Québec : Presses de l'Université Laval. Collection « Sociologie contemporaine ».

Vultur, M. et Trottier, C. (2010). Les trajectoires d'insertion professionnelle des jeunes Québécois diplômés de l'école secondaire. In Papinot, C. et Vultur, M. (dir.), Lesjeunesses au travail. Regards croisés France-Québec (p. 257-280). Québec : Presses de l'Université Laval. Collection « Regards sur la jeunesse du monde ».

Walther, A. (2006). Regimes of youth transitions. Choice, flexibility and security in young people's experiences across different European contexts. Young, 14 (2), 119-139.

\section{Notas}

1 Versión original publicada en francés con el título L'insertion professionnelle des jeunes : un concept historique, ambigu et sociétal en la Revue Jeunes et Societé, Volumen 1, número 2, 2016. La presente traducción del francés al español fue realizada por Cristina Vicente Lucas, alumna de Prácticas de Traducción del Traductorado Público Nacional en Lengua Francesa, FaHCE. UNLP. Tutora: Dra. Beatriz Cagnolati, profesora de Prácticas de Traducción y directora del Área de Investigación en Traductología/Laboratorio de Investigaciones en Traductología(AIT/LIT-IdIHCS-UNLP/ CONICET). Especialista de referencia: Dra. Mariana Busso, profesora FaHCE-UNLP e investigadora del Laboratorio de Estudios en Sociología y Economía del Trabajo (LESET- IdIHCS-UNLP/CONICET).

2 N de la T: El economista Jean Fourastié (1907-1990) es el creador de la expresión "Les Trente Glorieuses", título de su obra sobre el crecimiento excepcional de la segunda guerra mundial.

$3 \mathrm{~N}$ de la T: Recorrido [parcours, en francés]: En Francia el principal exponente de este enfoque fue Godard. El autor hace una diferenciación entre el concepto de recorrido y el de trayectoria. El recorrido es el encadenamiento de acontecimientos de la vida de un sujeto que pueden ser conocidos a través del relato del propio sujeto. Pero cuando analizamos trayectorias hacemos referencia a esquemas elaborados por el investigador en los que se incorporan los diferentes momentos de la vida de un sujeto. El análisis de trayectorias incorpora las "temporalidades sociales", es decir, analiza los distintos momentos de las trayectorias en un marco contextual.

$4 \mathrm{~N}$ de la T: Rapport, sustantivo de origen francés, polisémico, acción del verbo rapporter. En términos generales significa "traer de vuelta" o "crear una relación". Este concepto proviene del campo de la Psicología y es utilizado para referirse a la técnica de crear una conexión de empatía con otra persona, para que se comunique con menos resistencia.

5 Esta evolución puede llegar hasta crear de facto una obligación de inserción: "el sistema ayuda a la persona, pero, poco después, la castiga si ella no llega a transformar la ayuda limitada en el tiempo en un trampolín hacia la autonomía. Toda política de inserción francesa es así bifaz. El individuo obtiene una ayuda, pero esta se le retira, incluso si no alcanza a encontrar su autonomía" (Noblet, 2014, p. 15).

$6 \mathrm{~N}$ de la T: En Quebec, la enseñanza collégial corresponde a un nivel de formación que se da entre la enseñanza secundaria y la universitaria. En el sistema escolar quebequense, el collège es el primer lugar de formación no obligatorio y la elección de formación es personal. En el collégial, los programas de estudios preuniversitarios lindan con los programas de estudios técnicos. Los primeros preparan a los estudiantes universitarios, mientras que los segundos se conciben en función del mercado laboral. Sin embargo, la formación técnica puede también dar acceso a la universidad, a condición de que sean respetadas ciertas exigencias relacionadas con el programa de estudios universitarios elegido. 
$7 \mathrm{~N}$ de la T: La sociétépar actions simplifiée (SAS) [sociedad por acciones simplificada] es una sociedad comercial que ofrece a los accionistas una gran libertad de organización (definida por el estatuto). Esta sociedad está constituida por una o varias personas que solo deben responder por un máximo de sus aportes.

$8 \mathrm{Si}$ así fuera, se podría concluir que un estudiante que trabaja media jornada durante sus estudios es integrado laboralmente, lo que volvería a confundir la noción de participación en el mercado laboral con la de la inserción laboral.

9 Se inscribe de esta manera la invitación de Iribarne (1986) que sostiene que "las condiciones de inserción de los jóvenes [...] no pueden ser analizadas independientemente de los fundamentos de los sistemas educativos y de sus relaciones institucionales, por un lado, con las empresas y por otro, con los sindicatos de trabajadores" (Iribarne, 1986, p. 95).

10 "Transitions between youth and adulthood are structured by a complex system of socio-economic structures, institutional arrangement and cultural patterns" (Walther, 2006, p.124).

11 Un acuerdo nacional interprofesional celebrado por las organizaciones patronales y sindicales entiende lo esencial de sus disposiciones adoptadas por una ley, tanto así que en ciertos textos, el Estado fue cualificado de "secretario judicial de los agentes sociales" (Méhaut, 2006, p. 39). En este ámbito, el proceso se inauguró con la ley de 1971 sobre "la formación profesional en el marco de la educación permanente" y continuado regularmente: es así que un acuerdo de 1983 sobre las formaciones en alternancia fue reanudado en una ley de 1984 que instaura particularmente, para los jóvenes de menos de 26 años, los contratos de cualificación.

$12 \mathrm{~N}$ de la T: Lecontrat de professionnalisation [contrato de profesionalización] es un contrato de trabajo que se da en Francia, celebrado entre un empleador y un empleado. Permite la adquisición -en el marco de formación continuauna cualificación profesional (diploma, título, certificado de calificación profesional...) reconocida por el Estado y/o la rama profesional. El objetivo es la inserción o el regreso al empleo de los jóvenes y adultos.

13 Sarfati (2014) destaca la diversidad de los usos a los que puede estar sujeto un dispositivo de alternancia en la educación superior en función particularmente de los proyectos personales de los jóvenes.

14 En este aspecto, Lochet (2003) muestra claramente que para los empleadores, la noción de "principiante" difiere de la del que "sale del sistema educativo" utilizada por las encuestas Génération [Generación]; en efecto estos hacen referencia a la noción de experiencia para calificar, con respecto a sus propias referencias que varían según el tipo de gestión del trabajo ejecutado. Algunos valoran significativamente las experiencias adquiridas en periodo de estudios.

15 En el caso de Francia, si desde el punto de vista de las convenciones estadísticas, esta constatación sigue siendo verdadera, desde el punto de vista práctico, la impenetrabilidad ha resultado afectado por el desarrollo de la alternancia escuela/ trabajo y por combinación de empleo/estudios que se desarrollan en los estudiantes. Véase al respecto Bordigoni y Vergnies (1997), al igual que los resultados de la última encuesta "Génération” [Generación] sobre los que salen del sistema educativo del 2010 interrogados en el 2013 (CÉREQ, 2014).

16 Solo quedaría por documentar sus lazos con la clásica noción de recorrido de vida.

$17 \mathrm{~N}$ de la T: La sécurisation, neologismo francés cuya traducción al español aún está en discusión. Según la Direction de l'animation de la recherche, des études et des statistiques DARES [Dirección de la organización de investigación, estudios y estadísticas], la idea de sécurisation invita a conciliar dos enfoques: el primero (preventivo) busca dar a los individuos un mejor control de su futuro profesional, hoy en día moldeado por la herencia académica que reproduce, muy ampliamente, las irregularidades sociales. El segundo enfoque, más curativo, busca atenuar lo más posible las consecuencias de los avatares de la vida profesional, particularmente favoreciendo una inserción o una reinserción de la manera más rápida posible.

18 Esta implantación política se realizó con la mediación de cuatro acuerdos interprofesionales; el título del último data del 7 de enero del 2009, trata sobre "el desarrollo de la formación a lo largo de la vida profesional, la profesionalización y la sécurisation de los recorridos laborales”, el término recorrido aparece 48 veces en el texto (Mériaux, 2009, p. 14).

19 Esta cuestión fue recientemente objeto de un coloquio titulado: "la sécurisation de los recorridos laborales: la situación en Quebec”, organizado en marzo del 2016 en la Université Laval par l'Alliance de recherche universités-communautés (ARUC) Innovations travail et emploi [Universidad Laval por la Alianza de investigación universidades-comunidades Innovaciones trabajo y empleo].

20 Véase particularmente Flamand (2016) en el marco de "Francia estrategia".

$21 \mathrm{~N}$ de la T: La capabilité (traducción del neologismo capability) se considera a partir de las posibilidades concretas que tienen los individuos de utilizar los bienes para transformarlos en acción real o, para retomar otra noción de Sen, en "accomplissement" (achievement) (Nay Olivier. Amartya Sen, Repenser l'inégalité. In: Revue française de science politique, $51^{\mathrm{e}}$ année, ${ }^{\circ} 1-2,2001$. pp. 311-315). 